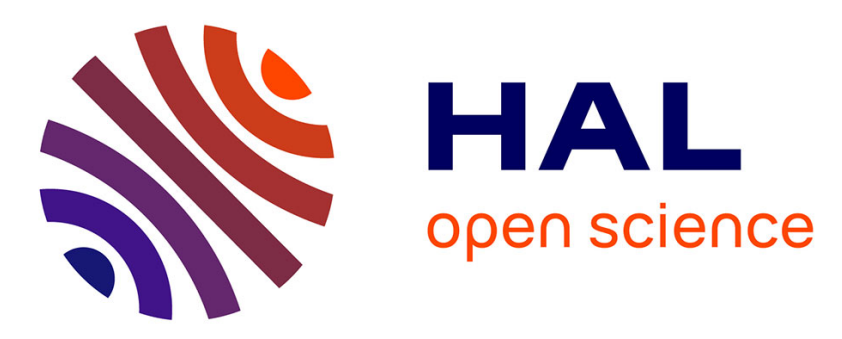

\title{
A robotic control framework for 3-D quantitative ultrasound elastography
}

Pedro A Patlan-Rosales, Alexandre Krupa

\section{To cite this version:}

Pedro A Patlan-Rosales, Alexandre Krupa. A robotic control framework for 3-D quantitative ultrasound elastography. IEEE Int. Conf. on Robotics and Automation, ICRA'17, May 2017, Singapore, Singapore. hal-01481975

\section{HAL Id: hal-01481975 \\ https://hal.inria.fr/hal-01481975}

Submitted on 3 Mar 2017

HAL is a multi-disciplinary open access archive for the deposit and dissemination of scientific research documents, whether they are published or not. The documents may come from teaching and research institutions in France or abroad, or from public or private research centers.
L'archive ouverte pluridisciplinaire HAL, est destinée au dépôt et à la diffusion de documents scientifiques de niveau recherche, publiés ou non, émanant des établissements d'enseignement et de recherche français ou étrangers, des laboratoires publics ou privés. 


\title{
A robotic control framework for 3-D quantitative ultrasound elastography
}

\author{
Pedro A. Patlan-Rosales ${ }^{1}$ and Alexandre Krupa ${ }^{2}$
}

\begin{abstract}
In this paper we present a novel approach to track and explore stiff tissues within 3-D ultrasound volumes acquired by a medical 3-D ultrasound probe mounted on a six degrees of freedom robotic arm. Autonomous palpation and on-line elastography process are implemented to estimate the elastic property of the tissues (strain) in a volume of interest (VoI) indicated by the user. The compression motion, required for the elastography, is performed by controlling the force applied by the ultrasound probe to the tissues. A visual servoing control for centering a rigid tissue (target) inside the field of view (FoV) of the ultrasound probe is established to always maintain the target visible. Additionally, rotations around the contact point between the tissue and the ultrasound probe are teleoperated through a haptic device handled by the user in order to allow exploration of the target surrounding areas. Results show a stable system that can be used in the future for diagnosis of diseases or tumor location.
\end{abstract}

\section{INTRODUCTION}

Ultrasound systems are one of the most common tools used in hospitals around the world. Besides its portability and low cost, it offers valuable information of tissues for doctors in real time. Radio frequency (RF) signals provided by an array of transducers are processed to display a bmode image. The properties of the RF signals can also be used to compute the elastic parameters of tissues, commonly known as strain values, over a region of interest (RoI). This procedure is called elastography, and it can be implemented by applying mechanical axial compression on the skin of the subject using the ultrasound probe. Elastography needs two arrays of RF signals successively measured at the pre- and post-compression states of the considered tissue to estimate its strain values along the probe axial direction.

In a previous work [1] we presented a three degrees of freedom (DoF) automatic palpation robotic system using force control and visual servoing to automatically position a 2-D US probe in a way to center on a stiff tissue based on the elastogram (map of strain values) in 2-D. Our approach was able to maintain the target in the center of the field of view (FoV) of a 2-D ultrasound probe even if perturbation motion inside the observation plane (in-plane motion) was applied to the system. However, this method did not consider motions outside the plane of observation of the 2-D probe (out-of-plane motions), as they are likely to occur in real examinations due to patient physiological

\footnotetext{
${ }^{1}$ Pedro A. Patlan-Rosales is with Inria Rennes-Bretagne Atlantique, Universite de Rennes 1, campus universitaire de Beaulieu, Rennes 35042, France. pedro-alfonso.patlan-rosalesdinria.fr

${ }^{2}$ Alexandre Krupa is with Inria Rennes-Bretagne Atlantique, campus universitaire de Beaulieu, Rennes 35042, France. alexandre.krupa@inria.fr
}

motions. Therefore, to deal with this issue, we propose in this paper to consider the elastograms in 3-D that we obtain by controlling the 6-DoF of a 3-D ultrasound probe with the new approach presented in section II.

Strain estimation in 3-D has been explored in different ways. In recent work [2], a method is presented using a linear probe mounted on a sliding mechanical track, estimating the elastograms in 2-D for a known number of positions resulting in an elastogram volume. In [3], a motorized 3-D probe has been used to estimate the 3-D strain map. In this previous work, a first sweep of the probe was performed to acquire the RF signals reflected by a volume of pre-compressed tissues. Then, a second probe sweep was performed to acquire the RF signals after an axial force on the consider volume of tissues was applied by the probe. The RF signals recorded before and after the tissues compression allow then to estimate the 3-D elastogram by dynamic programing. However, the procedure to obtain the elastograms in these works is performed manually, and it depends on the dexterity of the physician to move the ultrasound probe.

Recently, robotic systems have been designed to exploit the B-mode information generated by a 3D ultrasound. For example, the estimation of the deformation of an anatomical shape due to the physiological motions of a patient was presented in [4] based on the tracking of a deformable target modeled as a mesh. Another work that used the ultrasound information in 3-D was presented in [5] to automatically compensate the 3-D physiological motion of a subject thanks to the control of an US probe by visual servoing.

Up until now, ultrasound elastography has been used only in very few robot-assisted procedures. The surgical robot da Vinci (Intuitive Surgical Inc.) was employed to estimate the elastogram by performing an automatic palpation [6]. In this previous work, the motion of a laparoscopic ultrasound 2-D probe was controlled, and the elastograms, obtained after processing the acquired 2-D ultrasound data, were displayed to guide a minimal invasive surgery procedure. In a similar framework [7], an external mechanical excitation was applied instead of a palpation motion with the US probe. The mechanical excitator was positioned on the skin, and the laparoscopic ultrasound probe was displaced by teleoperating the da Vinci robot. However, this system lacks in the synchronization of the excitator and the US probe. In the same field of laparoscopy, a 11-DoF snake-like robot with an integrated phased ultrasonic micro array was used to locate hard lesions by palpation motion [8]. However, none of these works use directly the 3-D elastograms as control feedback for robotic tasks. We propose to extend 
our previous work to control the 6-DoF of a 3-D ultrasound probe. In order to allow elastography exploration in the 3-D space, we propose in this paper to maintain a target visible in the center of the 3-D FoV by visual servoing. This can help the physician to track a VoI regardless of the movement of the patient. We also propose to include in our control a teleoperation control mode for the rotations of the US probe thanks to the use of a haptic device. Teleoperating the probe rotations will therefore allow the clinician to explore in detail the surrounding areas of the VoI. To our knowledge, this is the first time that volumetric elastograms are used as control feedback for a robot.

We describe in the section II the methodology used for the automatic palpation, and the automatic centering of a rigid object in the $3-\mathrm{D}$ FoV. In the same section, we detail the teleoperation of the orientation of the ultrasound probe. Section III presents experimental results using an organic phantom. Then an argumentation and analysis of the results obtained from the experiments are presented in section IV.

\section{Methods}

\section{A. Automatic palpation}

We define autonomous palpation as a system to compute a strain map by compression motion with an ultrasound probe. A motorized ultrasound probe is used in this work (model 4DC7-3/40 Analogic Corporation) to obtain a volume composed by $N_{f}$ RF frames. Fig. 1 shows the cartesian reference frames attached to the 6-DoF robotic arm we used to move the probe. A force sensor (located at frame $\mathcal{F}_{s}$ in Fig. 1) is involved in the system to achieve the compression motion.

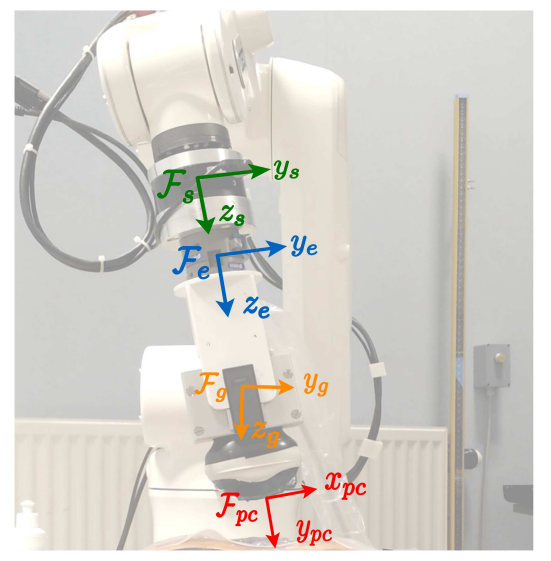

Fig. 1. Cartesian reference frames attached to the robotic arm.

As we previously show in the 2-D case [1], oscillatory force control was used to reach the desired compression motion. This task remains the same with respect to the control law. However, the period in the oscillatory force variation changes. The acquisition of one volume takes around one second, and we need the RF signals in the states of pre- and post-compression. Therefore, a step-variation function proposed for the force changing is expressed as follows:

$$
F_{d}(\tau)= \begin{cases}F_{\max } & \text { if } \tau \text { is odd } \\ F_{\min } & \text { otherwise }\end{cases}
$$

where $\tau \in \mathbb{Z}^{+}$increases every acquisition of one volume. $F_{\min }$ and $F_{\max }$ are the forces applied for pre- and postcompression respectively. These force values depend in the tissue stiffness.

The force/torque sensor provides the measures for the force tensor ${ }^{s} \mathbf{H}_{s}$ expressed in the sensor frame $\mathcal{F}_{s}$. As we need to measure the force applied in the contact point frame $\mathcal{F}_{p c}$, we are taking into account the probe mass $m_{p}$ in the gravity force tensor ${ }^{g} \mathbf{H}_{g}=\left[\begin{array}{lllllll}0 & 0 & 9.81 m_{p} & 0 & 0 & 0\end{array}\right]^{\top}$. The tensor used to express the force measured in $\mathcal{F}_{p c}$ is defined as follows [9]:

$$
{ }^{p c} \mathbf{H}_{p c}={ }^{p c} \mathbf{F}_{s}\left({ }^{s} \mathbf{H}_{s}-{ }^{s} \mathbf{F}_{g}{ }^{g} \mathbf{H}_{g}\right)
$$

where ${ }^{s} \mathbf{F}_{g}$ and ${ }^{p c} \mathbf{F}_{s}$ are transformation matrices from the gravity frame $\mathcal{F}_{g}$ to the frame $\mathcal{F}_{s}$ and from the frame $\mathcal{F}_{s}$ to the frame $\mathcal{F}_{p c}$ respectively.

In our case, we are aiming to control the force in the $y$-axis of the probe. Then, we define the feature vector to be regulated as $\mathbf{s}_{f}=\left[\begin{array}{llllll}0 & 1 & 0 & 0 & 0 & 0\end{array}\right]^{p c} \mathbf{H}_{p c}$ and the desired feature vector as $\mathbf{s}_{f}^{*}=\left[\begin{array}{lllllll}0 & F_{d}(\tau) & 0 & 0 & 0 & 0\end{array}\right]$. The error to minimize is $\mathbf{e}_{f}=\mathbf{s}_{f}-\mathbf{s}_{f}^{*}$, with an exponential decrease of $\mathbf{e}_{f}$. Therefore, the desired variation of the error is defined as $\dot{\mathbf{e}}_{f}^{*}=-\lambda_{f} \mathbf{e}_{f}$ with $\lambda_{f}$ being the force control gain. The change of the force feature through time can be expressed as $\dot{\mathbf{s}}_{f}=\mathbf{L}_{f} \mathbf{v}$ where $\mathbf{L}_{f}$ is the interaction matrix that relates the force feature to the probe velocity $\mathbf{v}=\left[\begin{array}{llllll}v_{x} & v_{y} & v_{z} & \omega_{x} & \omega_{y} & \omega_{z}\end{array}\right]^{\top}$ with the three first components corresponding to the translational velocity and the three last components to the angular velocity expressed in the contact point frame $\mathcal{F}_{p c}$ (see Fig. 1). Then $\mathbf{L}_{f}$ can be approximated as $\mathbf{L}_{f}=\left[\begin{array}{llllll}0 & k & 0 & 0 & 0 & 0\end{array}\right]$, where $k$ is an estimation of the contact stiffness. The control law for this task is defined as

$$
\mathbf{v}_{f}=\mathbf{L}_{f}^{+} \dot{\mathbf{e}}_{f}^{*}
$$

where $\mathbf{L}_{f}^{+}$is the Moore-Penrose pseudo-inverse of $\mathbf{L}_{f}$.

\section{B. Estimation and post-processing of the 3-D elastogram}

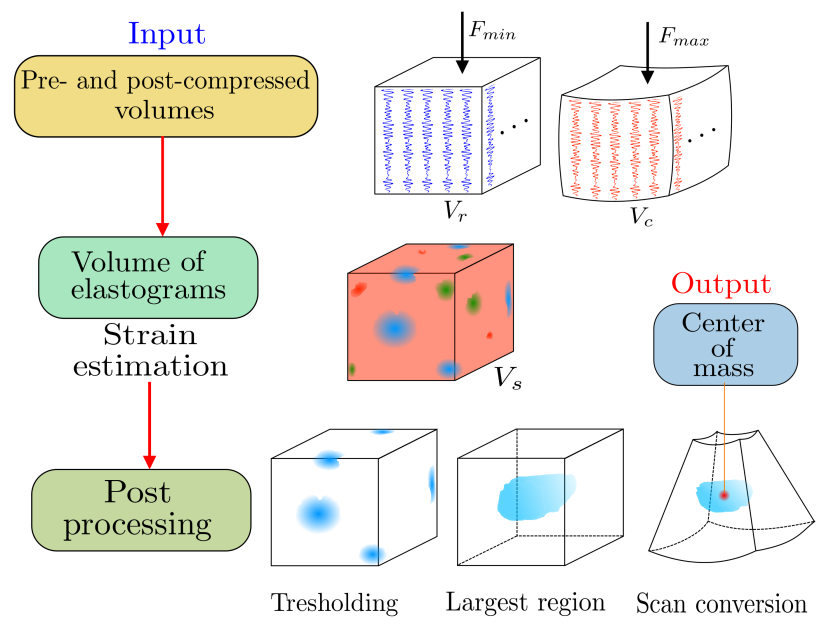

Fig. 2. Proposed process to obtain the center of mass of the largest stiff region.

In Fig. 2 we show the process to estimate the center of mass of the biggest rigid tissue. We use the volumes acquired 
with RF signals (RF volumes) $V_{r}$ and $V_{c}$ in the pre- and postcompression motion respectively to compute a $3-\mathrm{D}$ strain volume into a VoI. We define the 3 -D strain volume $V_{s}$ as

$$
V_{s}(i, j, k)=A_{s_{k}}(i, j)
$$

where $i$ and $j$ are the indexes of the ultrasound scanline and the sample in the scanline respectively. $A_{s_{k}}$ is the strain map in 2-D estimated at the $k$-frame pair of $V_{r}$ and $V_{c}$ in the interval $\left[k_{0}, k_{n}\right]$ such that $k_{n}>k_{0}$. The elastograms in 2-D are approximated using the method presented in [1], which are based on the optical flow for the motion estimation between the pre- and post-compression 2-D RF frames and the least-squares strain estimator [10].

The obtained $V_{s}$ volume provides elastic information of the tissues inside the VoI as we show in Fig. 2, where the red color represents the soft tissue and the blue the stiff tissue. Furthermore, we propose to segment $V_{s}$ using a threshold between $-\mu$ and $\mu$, to isolate the stiff tissues. We estimate $\mu=c_{s}+p_{s} \max \left(\left\|V_{s}\right\|\right)$, where $c_{s}=\min \left(V_{s}\right)+$ $0.5\left[\max \left(V_{s}\right)-\min \left(V_{s}\right)\right]$ is the central strain value, $p_{s}$ is the percentage of the strain values to use and $\|\star\|$ is the absolute value of $(\star)$. The value of $p_{s}$ allows to restrict the limits of the thresholding for the strain values. As we want to detect only the rigid tissues, the value of $p_{s}$ should be low, and we have found experimentally that $p_{s}=0.1(10 \%)$ gives us the best results. The thresholding provides the regions with only stiff tissue. We detect and track the biggest region in order to keep it always visible as described in subsection II-C. To compute the biggest region and its center of mass (CoM), we apply the connected components algorithm in 3-D to $V_{s}$ after thresholding. In our case, we use the implementation of this algorithm provided by the VTK library (Visualization Toolkit [11]) with the function vtkPolyDataConnectivityFilter to obtain the largest stiff region $V_{s b}$ and the vtkCenterOfMass function to calculate the center of mass $\left(\mathbf{p}_{c}=\left(i_{c}, j_{c}, k_{c}\right)\right)$ of $V_{s b}$. We show in Fig. 3 the mesh obtained to compute the center of mass.
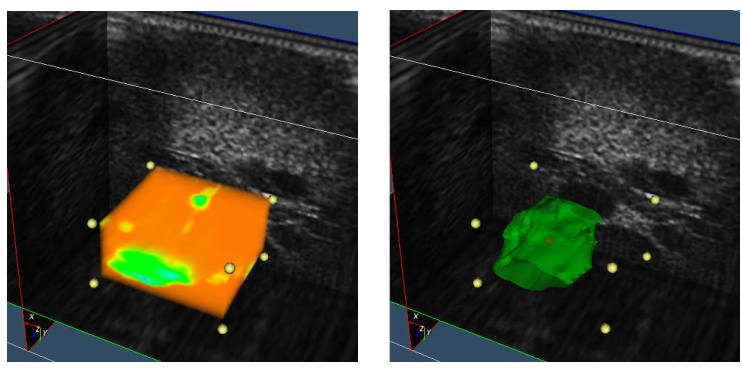

Fig. 3. Left image shows the volume of strains values $V_{s}$ (green color means stiff tissue and orange soft tissue). Right image shows the mesh of the biggest rigid tissue, $V_{s_{b}}$, in green.

We need to convert the value of $\mathbf{p}_{c}$ in the metric coordinates $\mathbf{p}_{g}$. To do this, as we use a convex ultrasound probe, we perform a scan conversion of each point inside the RF volume to the Cartesian coordinates (see Fig. 4), s $(i, j, k) \rightarrow$ $\mathbf{p}(x, y, z)$, in order to obtain the metric location with respect to the Cartesian frame. We define the scan conversion using the ultrasound probe parameters as described in [5]. In our case, RF data is considered instead of pre-scan images. We

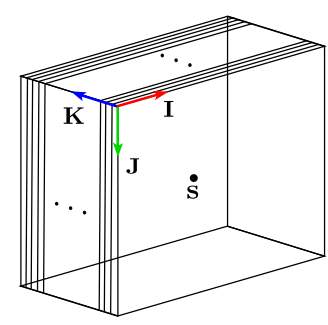

RF volume

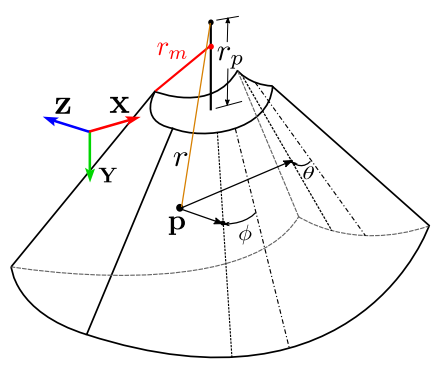

Cartesian volume
Fig. 4. Scan conversion in 3-D required to obtain the metric coordinate.

briefly recall the scan conversion formulation as

$$
\begin{aligned}
x & =r \sin \phi \\
y & =\left[r \cos \phi-\left(r_{p}-r_{m}\right)\right] \cos \theta+\left(r_{p}-r_{m}\right) \\
z & =\left[r \cos \phi-\left(r_{p}-r_{m}\right)\right] \sin \theta
\end{aligned}
$$

where $r_{p}$ and $r_{m}$ are the radii of the ultrasound probe and the motor of the probe respectively (see Fig. 4 right). The coordinates in the Cartesian volume are sorted as quasispherical coordinates with $r$ as the distance from the point to the origin of the scanlines, $\phi$ as the azimuthal angle in the $x-y$ plane and $\theta$ as the zenith angle (see Fig. 4 right). The quasi-spherical coordinates are computed in function of the RF coordinates as $r=\frac{v_{s}}{f_{s}} j+r_{p}, \phi=-0.5 \alpha_{l}\left(N_{f}-1\right)+\alpha_{l} i$ and $\theta=-0.5 \eta\left(N_{f}-1-2 k\right)$ where $v_{s}$ is the speed of the sound $(1540 \mathrm{~m} / \mathrm{s}), f_{s}$ is the sampled frequency, $\alpha_{l}$ is angle between neighboring scanlines and $\eta$ is the angle of the FoV of the motor in the ultrasound probe for a motor angular step.

Every slice function of $V_{r}, f_{k}(i, j) \in V_{r}$, is converted to b-mode (brightness mode), $f_{k_{b}}(i, j)$, image as $f_{k_{b}}=$ $\log \left\{\|\right.$ Hilbert $\left.\left(f_{k}\right) \|\right\}$ where Hilbert $(\star)$ is the Hilbert transform of $(\star)$. For visualization purposes, images are normalized from 0 to 255 , and the scan conversion previously described is applied. We display one volume every two volumes due to the computational cost. The display rate is two times the period of the volume acquisition $T_{v}$. We also display $V_{s b}$ with a mesh (using marching cubes mesh in VTK) at the same display rate as shown in Fig. 3.

\section{Target-probe centering on $x-z$ plane}

Keeping a target in the field of view (FoV) of the volume of analysis is a task which requires the displacement of the ultrasound probe on the $x-z$ plane (see Figure 5). The center of mass of the target, $\mathbf{p}_{g}=\left(x_{g}, y_{g}, z_{g}\right)$ (estimated in the section II-B), comes from the automatic palpation. As we control the probe velocity in $y$-axis with the automatic palpation, the remaining translational velocities $v_{x}$ and $v_{z}$ can therefore be controlled by visual servoing to perform an automatic alignment of the target in the middle of the probe FoV.

The visual feature vector that we define to perform this task is $\mathbf{s}_{t}=\left[\begin{array}{ll}x_{g} & z_{g}\end{array}\right]^{\top}$. In this case the desired visual feature vector to reach the centering of the object of interest in the probe FoV is directly $\mathbf{s}_{t}^{*}=\mathbf{0}_{2 \times 1}$. The error is defined as $\mathbf{e}_{t}=\mathbf{s}_{t}-\mathbf{s}_{t}^{*}$. Similar to the force control designed for the automatic palpation task, an exponential decrease of the error can be obtained by defining the desired error variation as 


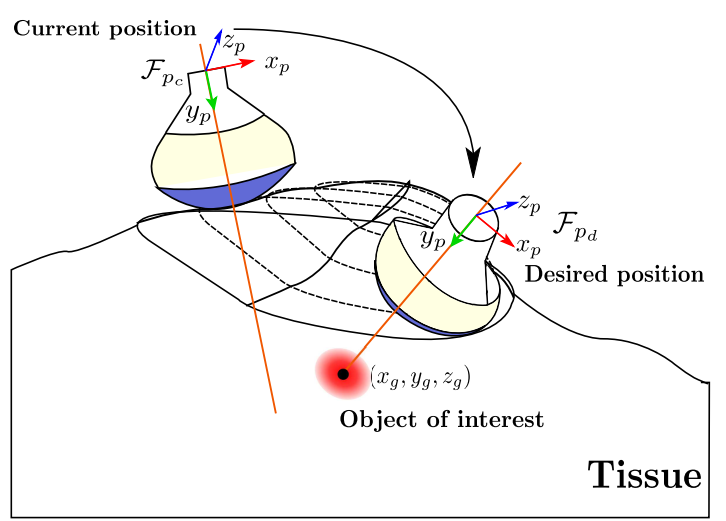

Fig. 5. Probe motion in the space from the current position to the desired position.

$\dot{\mathbf{e}}_{t}^{*}=-\lambda_{t} \mathbf{e}_{t}$ with $\lambda_{t}$ being the target-probe centering control gain.

Using basic kinematics, we determine the relation between the probe velocity $\mathbf{v}$ and the variation of the retained features as

$$
\left[\begin{array}{c}
\dot{x}_{g} \\
\dot{z}_{g}
\end{array}\right]=\left[\begin{array}{cccccc}
-1 & 0 & 0 & 0 & -z_{g} & y_{g} \\
0 & 0 & -1 & -y_{g} & x_{g} & 0
\end{array}\right] \mathbf{v}
$$

The Equation (8) can be written as $\dot{\mathbf{s}}_{t}=\mathbf{L}_{t} \mathbf{v}$, where $\mathbf{L}_{t}$ is the interaction matrix related to $\mathbf{s}_{t}$. Then, the control law for the target-probe centering can be expressed as

$$
\mathbf{v}_{t}=\mathbf{L}_{t}^{+} \dot{\mathbf{e}}_{t}^{*}
$$

\section{Teleoperation of probe orientation}

The third task we propose consists in teleoperating the probe orientation in order to explore with detail the surrounding area of the target. To perform this, we define the orientation of the probe as reaching a desired orientation $\mathbf{s}_{\theta}^{*}=\left[\begin{array}{lll}\theta_{x_{d}} & \theta_{y_{d}} & \theta_{z_{d}}\end{array}\right]^{\top}$ from the measure feature vector $\mathbf{s}_{\theta}=\left[\begin{array}{lll}\theta_{x} & \theta_{y} & \theta_{z}\end{array}\right]^{\top}$, where $\theta_{x}, \theta_{y}, \theta_{z}$ are the measured angles of the ultrasound probe provided by the robot odometry at the current time $t_{c}$. The error to minimize is defined as $\mathbf{e}_{\theta}=\mathbf{s}_{\theta}-\mathbf{s}_{\theta}^{*}$, and the desired exponential error decrease can be achieved by the desired error variation expressed as $\dot{\mathbf{e}}_{\theta}^{*}=-\lambda_{\theta} \mathbf{e}_{\theta}$ where $\lambda_{\theta}$ is the orientation control gain.

As in the previous task, we determine the interaction matrix that relates the feature vector variation, $\dot{\mathbf{s}}_{\theta}$, with the probe's velocity $\mathbf{v}$ as

$$
\mathbf{L}_{\theta}=\left[\begin{array}{cccc} 
& -1 & 0 & 0 \\
\mathbf{0}_{3 \times 3} & 0 & -1 & 0 \\
& 0 & 0 & -1
\end{array}\right]
$$

The control law for the probe orientation is then provided by

$$
\mathbf{v}_{\theta}=\mathbf{L}_{\theta}^{+} \dot{\mathbf{e}}_{\theta}^{*}
$$

In our case we propose to set and change on demand the desired probe orientation $\mathbf{s}_{\theta}^{*}$ by manually moving the three rotational degrees of freedom of a haptic device (Haption VIRTUOSE 6D). Using the odometry of the haptic device, we obtain a reference orientation $\boldsymbol{\Phi}_{\text {init }}$ at the time $t_{0}$ and the current orientation $\boldsymbol{\Phi}_{c}$ at the current time $t_{c}$ when the endeffector is moved. Then, we express the desired orientation as

$$
\mathbf{s}_{\theta}^{*}={ }^{p c} \mathbf{R}_{v}\left(\boldsymbol{\Phi}_{c}-\boldsymbol{\Phi}_{\text {init }}\right)
$$

where ${ }^{p c} \mathbf{R}_{v}$ is the rotation matrix between haptic frame $\mathcal{F}_{v}$ and the probe contact frame $\mathcal{F}_{p c}$.

\section{E. General control law}

Our goal is to combine the automatic palpation with the alignment of the probe on the target and the teleoperation of the rotation of the probe. Therefore, to achieve our goal, we enclose the previous control laws into a general control law by stacking the interaction matrices $\mathbf{L}_{f}, \mathbf{L}_{t}$ and $\mathbf{L}_{\theta}$ of each task in a general interaction matrix. This latter relates the variation of the features of the three tasks to the 6-DoF probe velocity, and it is obtained as follow

$$
\mathbf{L}=\left[\begin{array}{cccccc}
-1 & 0 & 0 & 0 & -z_{g} & y_{g} \\
0 & k & 0 & 0 & 0 & 0 \\
0 & 0 & -1 & -y_{g} & x_{g} & 0 \\
0 & 0 & 0 & -1 & 0 & 0 \\
0 & 0 & 0 & 0 & -1 & 0 \\
0 & 0 & 0 & 0 & 0 & -1
\end{array}\right]
$$

Finally, the general control law that allows to perform simultaneously the three tasks is given by

$$
\mathbf{v}=\mathbf{L}^{+}\left[\begin{array}{c}
-\lambda_{t_{0}} e_{t_{0}} \\
-\lambda_{f} e_{f} \\
-\lambda_{t_{1}} e_{t_{1}} \\
-\lambda_{\theta} \mathbf{e}_{\theta}
\end{array}\right]
$$

where $-\lambda_{t_{0}} e_{t_{0}}$ and $-\lambda_{t_{1}} e_{t_{1}}$ represent the first and the second elements of $\dot{\mathbf{e}}_{t}^{*}$ respectively.

The tasks of centering the target in the FoV and the probe orientation require low gain to converge successfully due to dependency of the time to retrieve a new RF volume in order to compute the center of mass. However, if we apply adaptive gain for these tasks, we can decrease the convergence time for these tasks. Then, adaptive gain is applied to $\lambda_{\mathbf{t}}$ and $\lambda_{\theta}$, and it is defined as follows

$$
\lambda(x)=a e^{-b x}+c
$$

where $a, b$ and $c$ are constant parameters and $x=\left\|\mathbf{s}^{*}-\mathbf{s}\right\|$ is the input to consider. The desired feature vector $\mathbf{s}^{*}$ and the measured feature vector $\mathbf{s}$ are linked to the corresponding vectors in the task where the adaptive gain is applied. The parameters $a, b$ and $c$ are computed as follows

$$
\begin{aligned}
a & =\lambda(0)-\lambda(\infty) \\
b & =\dot{\lambda}(0) / a \\
c & =\lambda(\infty)
\end{aligned}
$$

where $\lambda(0)$ is the gain when $x=0, \lambda(\infty)$ is the gain when $x=\infty$ and $\dot{\lambda}(0)$ is the slope of $\lambda(x)$ when $x=0$.

\section{EXPERIMENTAL RESULTS}

For the experiments we used a 6-DoF robot, Viper s850 (Adept Technology Inc., USA) and an Analogic Corporation 4DC7-3/40 convex ultrasound probe rigidly attached to its end-effector. The force sensor used was an ATI Gamma 65-SI FT connected to a National Instrument NI DAQmx PCI-6220 board. The acquisition of RF data was implemented using a server-client TCP/IP communication in a local network. 
We used as server the SonixTouch ultrasound scanner, and as client a workstation (Intel Xeon CPU @2.1 GHz) that performs all the imaging process, control law computation and communication with the robot. The RF data from the server is sent to the client at the rate of 24 FPS (frames per second). Every volume contains $31 \mathrm{RF}$ frames which means that every volume is completed in $\simeq 1.3 \mathrm{~s}$. In the client, we developed a multi-thread program in $\mathrm{C}++$, with a graphic user interface (GUI) in the main thread created to display and control the functions of the other threads, as described in Fig. 6. A shared pointer is continuously updated by the acquisition thread (frame by frame). This shared pointer is read by the RFtoBMode thread (process in charge of converting the RF volume to b-mode volume) and the Elastography thread (process to compute the 3-D elastogram in a VoI) once a volume is completed. The Display object, in the main thread, contains the functions to display three orthogonal planes (sagittal, axial, coronal) of the volume (see Fig 7). This object also allows the user to select the VoI by displacing the planes to the desired position and pressing a key of the keyboard.

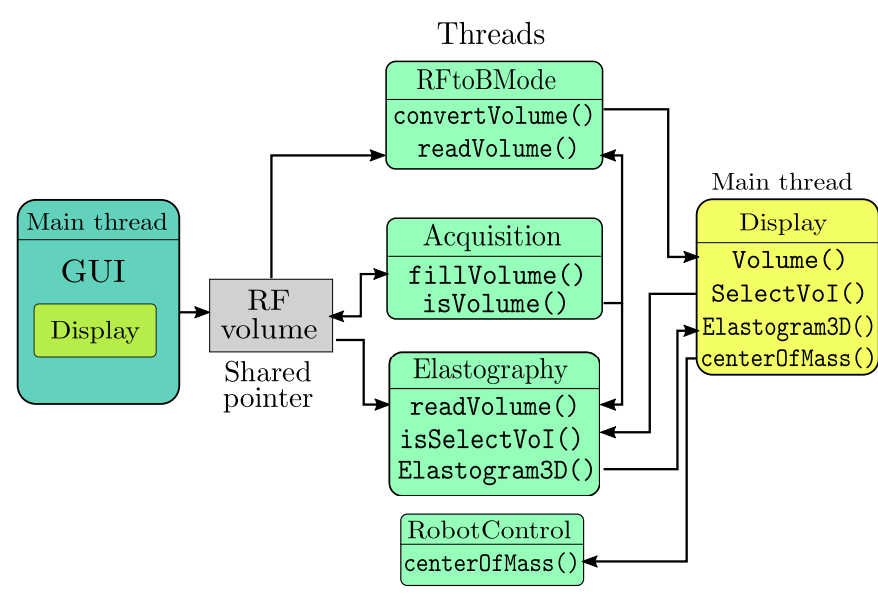

Fig. 6. Short diagram of the implemented multithread program.

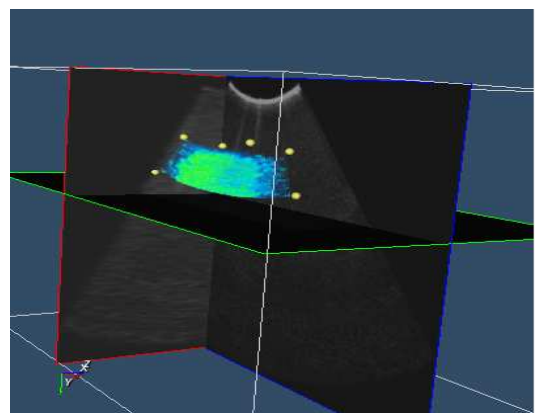

Fig. 7. Display of the three orthogonal planes and the VoI with the 3D elastogram.

We perform a set of experiments on a homemade gelatin phantom with two duck gizzards inside (see Fig. 8), and we present in this paper only the evolution of one experiment, which is the base for every experiment performed. We set the values of $F_{\min }=1.5 \mathrm{~N}, F_{\max }=2.5 \mathrm{~N}$ and $\lambda_{f}=$ 0.002. $F_{\min }$ and $F_{\max }$ were estimated empirically for the gelatin phantom, and they can be adapted for any other kind of tissue. The parameters in the adaptive gains are set as $\lambda_{t}(0)=0.1, \lambda_{t}(\infty)=0.03, \dot{\lambda}_{t}(0)=0.3, \lambda_{\theta}(0)=1.5$, $\lambda_{\theta}(\infty)=0.2$ and $\dot{\lambda}_{\theta}(0)=2.3$. The experiment begins with an initial probe position where a stiff object of interest is positioned in the 3-D US probe FoV (red point in Fig. 8left). Then, the automatic palpation with the robot is activated to perform the compression of the tissues. The centroid of the 3-D elastogram is estimated as we previously described in II-B, and the automatic centering control task is started. Next, four points delimiting the $\mathrm{VoI}$ are chosen using the developed GUI and displayed by small yellow spheres as shown in Fig 7. Once the $\mathrm{VoI}$ is selected, the 3D elastogram is estimated for every pair of RF volumes in the same direction of acquisition. The centroid of the 3-D elastogram is estimated as we previously described in II-B, and it is sent to the automatic centering control task. The teleoperation of the probe orientation is always active, and the user can rotate the end-effector of the haptic device any time.

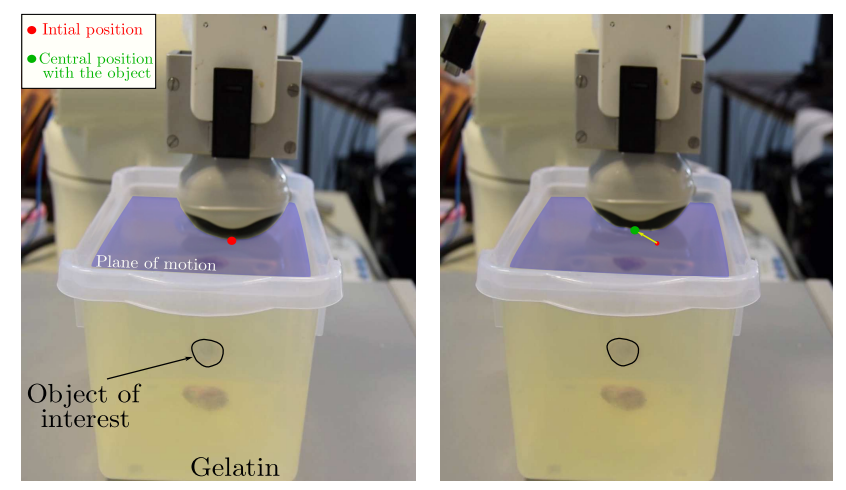

Fig. 8. Experiment with a gelatin phantom containing two duck gizzards.

We show in Fig. 9 the plots of the evolution of the probe velocities for one experiment. We can observe at the beginning of the experiment that the only velocity active is the $v_{y}$ (force control). At time $t \simeq 30 \mathrm{~s}$ the system is paused to select the VoI, and time $t \simeq 75 \mathrm{~s}$ the process continues. Then, the center of mass is computed and the velocities $v_{x}$ and $v_{z}$ start to variate, and at $t \simeq 150 \mathrm{~s}$ the probe has been automatically aligned with the stiff tissue of interest (duck gizzard) by following and exponential decrease of the visual error $\mathbf{e}_{t}$ as expected. The teleoperation of the probe orientation is introduced from the time $t \simeq 160 \mathrm{~s}$ and we can see at this point the variation of $\omega_{x}, \omega_{y}$ and $\omega_{z}$ with eventual convergence each time a new desired probe orientation is manually indicated by the user thanks to the haptic device.

\section{DISCUSION}

We observe that the process in the experiments requires fast acquisition of RF data sent by the ultrasound. However, the control of the probe is slow due to the requirement of the elastography of two volumes (pre- and post-compressed) to compute one 3-D elastogram. Therefore, if the gains for the automatic centering and for the probe orientation are set too high, these two tasks will fail due to the low volume rate acquisition. We also introduce small displacements to the gelatin when the system was running, and we observe that 


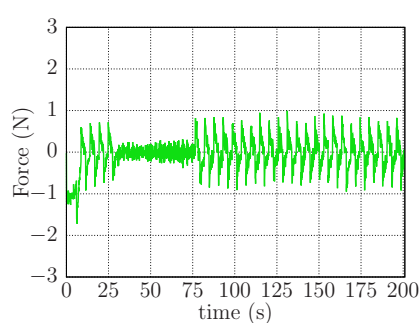

(a) Force error measured

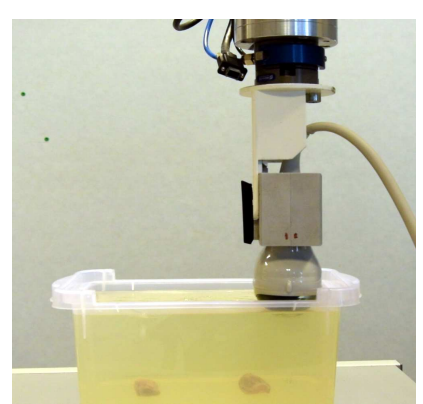

(e) Probe position before the VoI selection

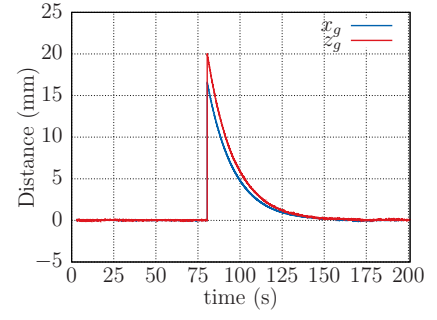

(b) Centering error $\mathbf{e}_{t}$

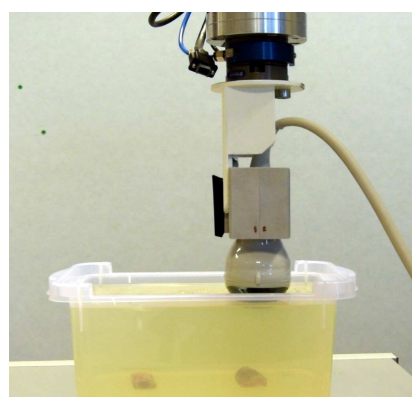

(f) Automatic alignment of the target in the FoV

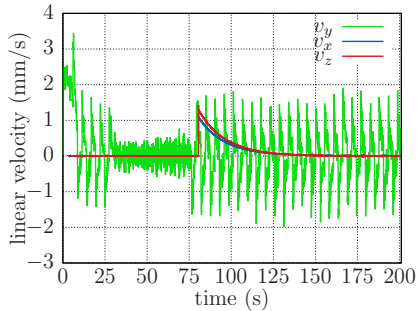

(c) Linear velocities

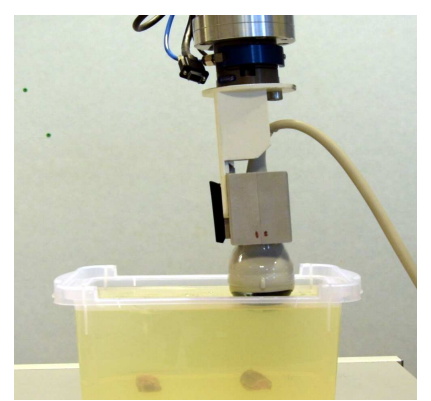

(g) Probe orientation by teleoperation

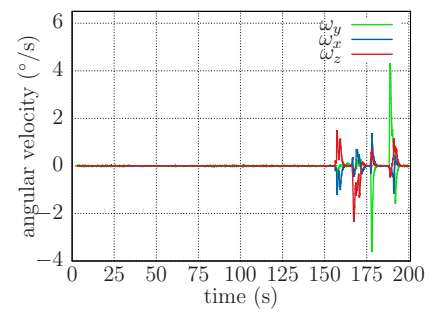

(d) Angular velocities

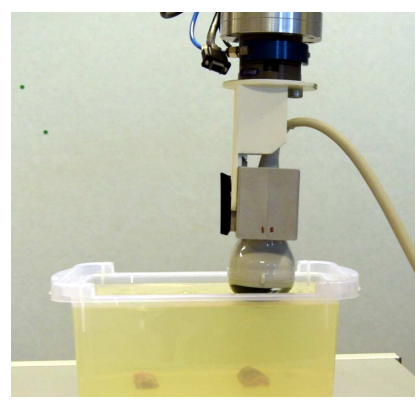

Fig. 9. Evolution through time of the reference experiment. (a) to (d) show the curves of the errors and velocities during the experiment. (e) to (g) show the pose of the ultrasound probe for centering of the object in the FoV and teleoperation of the probe orientation.

if the motions are slower than the time of the RF volume acquisition, then the process compensates successfully this perturbation. The main advantage of the system proposed in this work with respect to the 2-D [1] case is that the out-of-plane motions are considered. This represents a big step in the quest of medical robotic-assisted system that can help to improve medical procedures. Moreover, the proposed system allows the clinician to perform 3-D quantitative elastography by simply teleoperating the probe orientation without worrying on both the palpation motion and the target centering motion.

\section{CONClusion}

We present a framework to control a 3-D ultrasound probe held by a 6-DoF robotic arm in order to perform realtime 3-D quantitative ultrasound elastography. It is based on the design of a control law that simultaneously perform three tasks: autonomous palpation of tissues, probe automatic alignment on a stiff target of interest and teleoperation of the probe orientation. The experimental results demonstrated the feasibility of the proposed concept. In future work we plan to use the estimated strain volume to provide a feeling of the elasticity of the tissue to the user through the haptic device. Our system could also be used in other clinical scenarios as for example for real-time automatic estimation of tissue strain 3-D map required for robotic steering of flexible needle.

\section{ACKNOWLEDGMENT}

This work has been jointly supported by Inria Rennes Bretagne Atlantique and the National Council of Science and Technology of Mexico (CONACYT) under the Grant no. $242120 / 383765$.

\section{REFERENCES}

[1] P. A. Patlan-Rosales and A. Krupa, "Automatic palpation for quantitative ultrasound elastography by visual servoing and force control," in IEEE/RSJ Int. Conf. on Intelligent Robots and Systems, IROS'16, Daejeon, Korea, October 2016.

[2] Q. Huang, B. Xie, P. Ye, and Z. Chen, "3-D ultrasonic strain imaging based on a linear scanning system," IEEE Transactions on Ultrasonics, Ferroelectrics, and Frequency Control, vol. 62, no. 2, pp. 392-400, Feb 2015.

[3] H. Rivaz, I. Fleming, M. Matinfar, O. Ahmad, A. Khamene, M. Choti, G. Hager, and E. Boctor, "Ablation monitoring with a regularized 3D elastography technique," in 2008 IEEE Ultrasonics Symposium, Nov 2008, pp. 308-312.

[4] L. Royer, M. Marchal, A. Le Bras, G. Dardenne, and A. Krupa, "Realtime tracking of deformable target in $3 \mathrm{~d}$ ultrasound images," in IEEE Int. Conf. on Robotics and Automation, ICRA'15, Seattle, WA, May 2015, pp. 2430-2435.

[5] D. Lee and A. Krupa, "Intensity-based visual servoing for non-rigid motion compensation of soft tissue structures due to physiological motion using 4d ultrasound," in IEEE/RSJ Int. Conf. on Intelligent Robots and Systems, IROS'11, San Francisco, USA, September 2011, pp. 2831-2836.

[6] S. Billings, N. Deshmukh, H. J. Kang, R. Taylor, and E. M. Boctor, "System for robot-assisted real-time laparoscopic ultrasound elastography," vol. 8316, 2012, pp. $83161 \mathrm{~W}-83161 \mathrm{~W}-8$.

[7] C. Schneider, A. Baghani, R. Rohling, and S. Salcudean, "Remote ultrasound palpation for robotic interventions using absolute elastography," 2012, pp. 42-49.

[8] H. T. Sen, N. Deshmukh, R. Goldman, P. Kazanzides, R. H. Taylor, E. Boctor, and N. Simaan, "Enabling technologies for natural orifice transluminal endoscopic surgery (N.O.T.E.S) using robotically guided elasticity imaging," pp. 83161 Y-83 161Y-8, 2012.

[9] C. Nadeau and A. Krupa, "Intensity-based ultrasound visual servoing: Modeling and validation with 2-d and 3-d probes," Robotics, IEEE Transactions on, vol. 29, no. 4, pp. 1003-1015, Aug 2013.

[10] F. Kallel and J. Ophir, "A least-squares strain estimator for elastography," Ultrason Imaging, vol. 19, no. 3, pp. 195-208, Jul 1997.

[11] W. Schroeder, K. Martin, and B. Lorensen, Visualization Toolkit: An Object-Oriented Approach to 3D Graphics, 4th Edition, 4th ed. Kitware, December 2006. 